\section{Photodynamic therapy for retinal capillary hemangioma}

${ }^{1}$ Ocular Oncology Service, Moorfields Eye Hospital, London, UK

${ }^{2}$ Ocular Oncology Service, St Bartholomew's Hospital, London, UK

${ }^{3}$ Southampton Eye Unit, Southampton University General Hospital,

Southampton, UK

${ }^{4}$ Clinical and Experimental Sciences, University of Southampton, UK

Correspondence: Victoria Cohen, Ocular Oncology Service, St. Bartholomew's Hsopital, West Smithfield, London EC1A 7BE, UK.

Tel: + $44(0) 7540119314$;

Fax: + $44(0) 2076017863$

E-mail: Victoria.Cohen@

bartsandthelondon.nhs.uk

Received: 26 April 2012 Accepted in revised form: 30 October 2012

Published online: 4 January 2013

\begin{abstract}
Purpose To describe the results of photodynamic therapy (PDT) for juxtapapillary and peripheral retinal capillary hemangioma ( $\mathrm{RCH}$ ).

Patients and methods Interventional case series of four eyes (four patients) with juxtapapillary $\mathrm{RCH}$ and one eye (one patient) with peripheral $\mathrm{RCH}$. Two eyes with juxtapapillary RCH had received two sessions of full-fluence, double-duration PDT; whereas other two eyes had received single session of half-fluence, single-duration PDT. The peripheral RCH was treated with a single session of full-fluence, single-duration PDT. Results Two patients had von HippelLindau disease. Follow-up duration ranged from 4 months to 1 year. Pre-PDT visual acuity (VA) ranged from 20/200 to $\mathrm{HM}$ (juxtapapillary RCH) and 20/100 (peripheral $\mathrm{RCH})$. Among the eyes with juxtapapillary $\mathrm{RCH}$, tumor regression with partial resolution of macular edema was noted in two eyes (one eye each with half-fluence and full-fluence PDT), whereas two eyes had no change in tumor size with persistent macular edema. VA remained stable in three eyes and declined in one eye. In an eye with peripheral $\mathrm{RCH}$, regression of tumor and macular edema with VA improvement was noted. Post-PDT complications included epiretinal membrane (one eye) and transient exudative retinal detachment (one eye). Conclusion PDT can be effective in reducing macular edema associated with RCH but this does not always correspond with an improvement in VA especially for juxtapapillary tumors.

Eye (2013) 27, 438-442; doi:10.1038/eye.2012.259; published online 4 January 2013
\end{abstract}

Keywords: retinal capillary hemangioma; juxtapapillary retinal hemangioma; peripheral retinal hemangioma; hemangioblastoma;
VP Papastefanou ${ }^{1,2}$, S Pilli li , A Stinghe ${ }^{3}$, AJ Lotery ${ }^{3,4}$ and VML Cohen ${ }^{1,2}$

photodynamic therapy; von Hippel-Lindau disease

\section{Introduction}

Retinal capillary hemangioma (or hemangioblastoma; $\mathrm{RCH}$ ) is a benign vascular tumor, occurring either as an isolated tumor or as a component of the von Hippel-Lindau (VHL) disease. RCH can be found in the midperipheral retina, juxtapapillary retina, or at the intraneural portion of the optic nerve. Vision loss can occur either from exudation from the tumor causing intraretinal exudates and edema or by glial proliferation causing traction on the retina.

Various treatment options including observation, laser, cryotherapy, radiotherapy, and vitreoretinal surgery have been attempted based on the $\mathrm{RCH}$ variant; however, each of these treatment methods has limitations to their use and success rate. ${ }^{1}$ Studies using verteporfin photodynamic therapy (PDT) for both juxtapapillary and peripheral $\mathrm{RCH}$ have mixed anatomical and functional outcomes. ${ }^{2-11}$

Post-PDT side effects such as vascular occlusion, optic neuropathy, and tractional retinal detachment (RD) have been reported. More recently, intravitreal anti-vascular endothelial growth factor (VEGF) has been tried but the outcomes are variable. ${ }^{7,10,11}$ Currently, there is no evidence to suggest that PDT combined with anti-VEGF is superior to PDT alone.

As the optimal management of $\mathrm{RCH}$ remains debatable, we present our experience of treating five cases of $\mathrm{RCH}$ treated with PDT.

\section{Case reports}

Patients were evaluated at the Ocular Oncology Service, Moorfields Eye Hospital, London (ie, patients 1-4) and Southampton Eye Unit, Southampton (patient 5). Four eyes of four 
Table 1 Demographics, clinical features, and PDT treatment outcomes of five eyes with retinal capillary hemangioma

\begin{tabular}{|c|c|c|c|c|c|c|c|c|c|c|}
\hline Case & Age, sex & RCH subtype & PDT settings (s) & $\begin{array}{l}{ }^{\text {a }} P D T \\
\text { sessions }\end{array}$ & $\begin{array}{l}\text { Follow-up } \\
\text { duration } \\
\text { (months) }\end{array}$ & VA acuity outcome & $\begin{array}{l}\text { RCH elevation }{ }^{\mathrm{b}} \\
(\mathrm{mm})\end{array}$ & RCH status ${ }^{c}$ & $\begin{array}{l}\text { Macular } \\
\text { edema }^{c}\end{array}$ & Complication \\
\hline 1 & $21, \mathrm{M}$ & $\begin{array}{l}\text { Sessile, } \\
\text { peripapillary }\end{array}$ & $\begin{array}{l}\text { Standard } \\
\text { fluence } / 166 \mathrm{~s}\end{array}$ & 2 & 12 & Stable at $20 / 200$ & Stable at 1.5 & $\begin{array}{l}\text { Superficial } \\
\text { fibrosis }\end{array}$ & $\begin{array}{l}\text { Partially } \\
\text { resolved }\end{array}$ & None \\
\hline 2 & $32, \mathrm{M}$ & $\begin{array}{l}\text { Endophytic, } \\
\text { peripapillary }\end{array}$ & $\begin{array}{l}\text { Standard } \\
\text { fluence } / 166 \mathrm{~s}\end{array}$ & 2 & 21 & $\begin{array}{l}\text { Reduced } 20 / 120 \\
\text { to } 20 / 200\end{array}$ & Stable at 1.8 & No change & Persistent & None \\
\hline 3 & $42, \mathrm{~F}$ & $\begin{array}{l}\text { Sessile, } \\
\text { peripapillary }^{\mathrm{d}}\end{array}$ & Half fluence $/ 83 \mathrm{~s}$ & 1 & 4 & Stable at HM & Stable at 1.9 & No change & Persistent & None \\
\hline 4 & $52, \mathrm{~F}$ & $\begin{array}{l}\text { Endophytic, } \\
\text { peripapillary }\end{array}$ & Half fluence $/ 83 \mathrm{~s}$ & 1 & 26 & Stable at $\mathrm{CF}$ & $\begin{array}{l}\text { Reduced } 2.1 \\
\text { to } 1.3\end{array}$ & $\begin{array}{l}\text { Superficial } \\
\text { fibrosis }\end{array}$ & $\begin{array}{l}\text { Partially } \\
\text { resolved }\end{array}$ & ERM \\
\hline 5 & $32, \mathrm{~F}$ & $\begin{array}{l}\text { Endophytic, } \\
\text { peripheral }\end{array}$ & $\begin{array}{l}\text { Standard } \\
\text { fluence } / 83 \mathrm{~s}\end{array}$ & 1 & 9 & $\begin{array}{l}\text { Improved } \\
20 / 100 \text { to } 20 / 66\end{array}$ & Not available & Regressed & Resolved & $\begin{array}{l}\text { Exudative RD } \\
\text { and S/R } \\
\text { hemorrhage }\end{array}$ \\
\hline
\end{tabular}

Abreviations: ERM, epiretinal membrane; F, female; M, male; PDT, photodynamic therapy; $\mathrm{RCH}$, retinal capillary hemangioma; RD, retinal detachment;

S/R, sub-retinal; VA, visual acuity; VHL, von Hippel-Lindau.

${ }^{a}$ Number of PDT.

${ }^{\mathrm{b}}$ Tumor elevation on ultrasonography.

${ }^{\mathrm{c}}$ Post-PDT outcome.

${ }^{\mathrm{d}}$ VHL disease positive.

patients had juxtapapillary $\mathrm{RCH}$, whereas one eye of one patient had peripheral $\mathrm{RCH}$. All patients were treated with verteporfin PDT (Activis, Quantel Medical, Cournon $\mathrm{d}^{\prime}$ Auvergne, France) with a single spot covering the lesion using Area Centralis lens (Volk, Mentor, OH, USA). None of the patients had additional intravitreal injection of either triamcinolone acetonide or anti-VEGF.

Table 1 summarizes the patient demographics, clinical characteristics, and PDT treatment strategies and outcomes. Figure 1 shows the patient $2(a-d)$ with juxtapapillary $\mathrm{RCH}$, largely remaining unresponsive to two sessions of full-fluence, double-duration PDT and patient 4 (e-h) with juxtapapillary $\mathrm{RCH}$, which had partially responded to the single session of half-fluence PDT. Figure 2 illustrates patient 5 with peripheral $\mathrm{RCH}$ highlighting an exudative RD and sub-retinal hemorrhage (b) following PDT treatment, which resolved with time (c).

\section{Discussion}

In our series of five eyes with $\mathrm{RCH}$, two different PDT treatment protocols were used for eyes with juxtapapillary $\mathrm{RCH}$, ie., two eyes (patients 1 and 2) had received double-duration, full-fluence PDT, as done previously, ${ }^{2}$ whereas two eyes (patients 3 and 4 ) had received single-duration, half-fluence PDT. Retreatment was done taking under consideration ultrasonographic thickness or the presence of persistent sub-retinal fluid in the macula. This differential/variable PDT protocol did not influence the treatment outcome as one eye each from two protocols had partially resolution of macular edema after PDT treatment. Interestingly, both unresponsive patients were VHL positive. Patients 3 and 4 with juxtapapillary RCH received half-fluence PDT. The rationale for use of half-fluence PDT was to minimize the risk of ischemic optic neuropathy. ${ }^{2}$ Of the two patients treated with half-fluence PDT, patient 4 had a more favorable outcome than patient 3. Overall, PDT resulted in an improvement in macular edema in half of the patients with juxtapapillary RCH. However, this does not correspond with an improvement in visual acuity (VA). Singh $e a^{1}$ reported that VA rarely improved following treatment if the initial VA is poor $(<20 / 200)$.

Patient 5 was considered for PDT as it could offer better penetration and reduce the likelihood of extensive hemorrhage. Following treatment, patient had transient exudative RD with an area of sub-retinal hemorrhage and increase in serous detachment of the fovea at 1 week post PDT, possibly representing a reactionary edema to $\mathrm{PDT}, 6,9,12$ resulting from change in tumor itself or due to breakdown of blood-retinal barrier. Despite this, there was no exaggerated cicatrical response in our case as observed by other authors. ${ }^{8}$ This case is probably representative of a better anatomical and functional outcome following PDT. Though peripheral $\mathrm{RCH}$ is thought to be more amenable to treatment due to its peripheral location, complications, such as epiretinal membrane formation and tractional RD can occur.

Anti-VEGF agents were not used in the management of these cases. Even though the VHL-related tumors are thought to be VEGF dependent, these tumors failed to show a consistent response to anti-VEGF therapy, both in the form of monotherapy or in combination with PDT. 70,11 Thus, the efficacy of the anti-VEGF therapy remains unclear. Although several small studies have described the use of PDT for $\mathrm{RCH}$, the exact reason for 

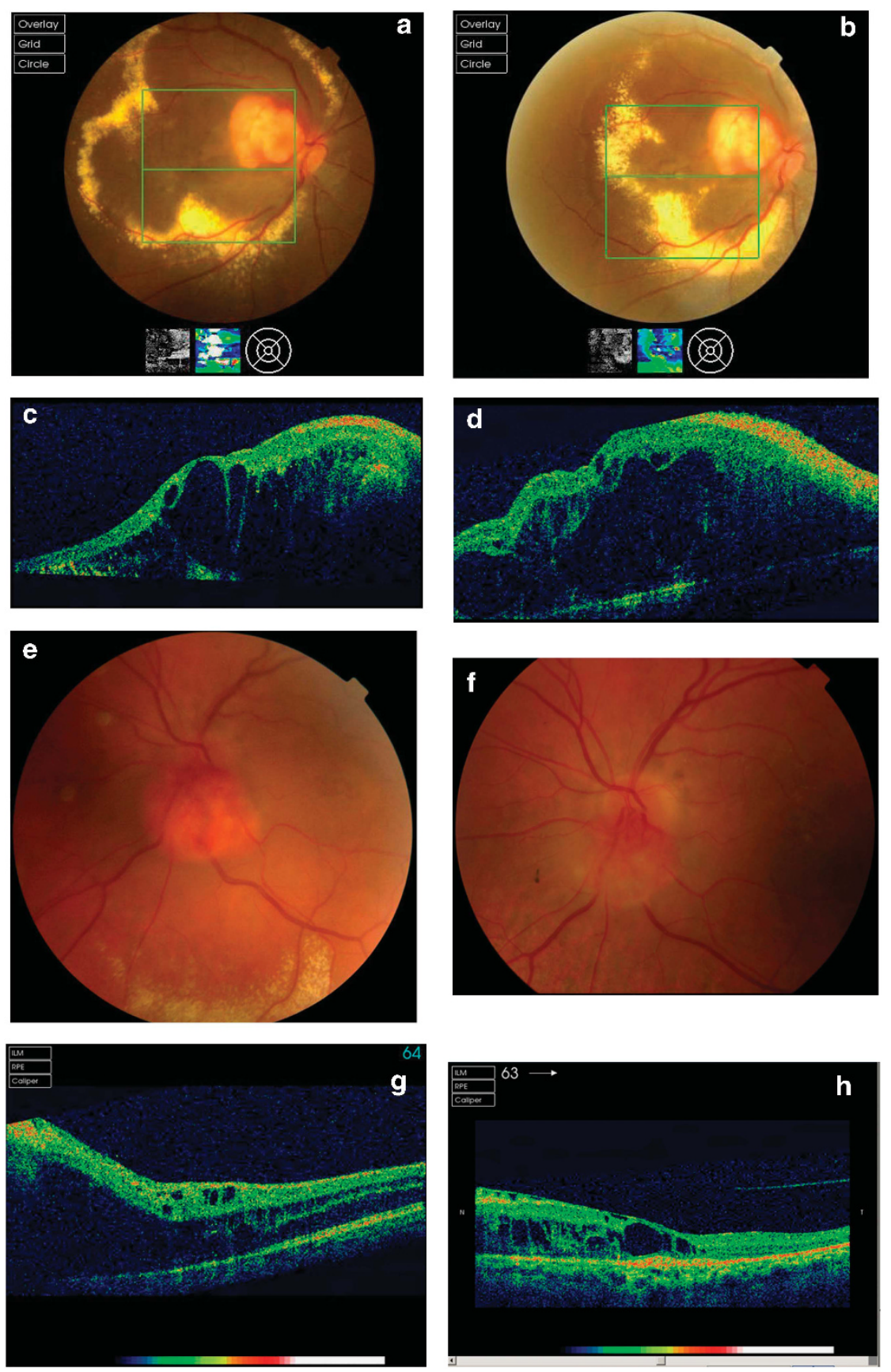

Figure 1 Color fundus photographs (CF) and spectral domain optical coherence tomography (SDOCT) foveal B scans of the right eye of a 32-year-old male (patient 2; a-d) and of a 52-year-old female patient (patient 4). (a) CF photograph of the posterior pole shows a juxtapapillary capillary hemangioma (before PDT) with associated exudative RD. (b) CF photograph shows no change in the capillary hemangioma (21 months after PDT). (c) SDOCT foveal B scan shows macular RD (before PDT). (d) SDOCT foveal B scan shows no significant change (21 months after PDT). (e) CF photograph of the posterior pole shows a juxtapapillary capillary hemangioma overlying the optic nerve (before PDT). (f) CF photograph of the posterior pole shows significant reduction and fibrosis of the capillary hemangioma (26 months after PDT). (g) SDOCT foveal B scan shows macular RD (before PDT). (h) SDOCT foveal B scan shows partial resolution of macular edema with an epiretinal membrane (26 months after PDT). 

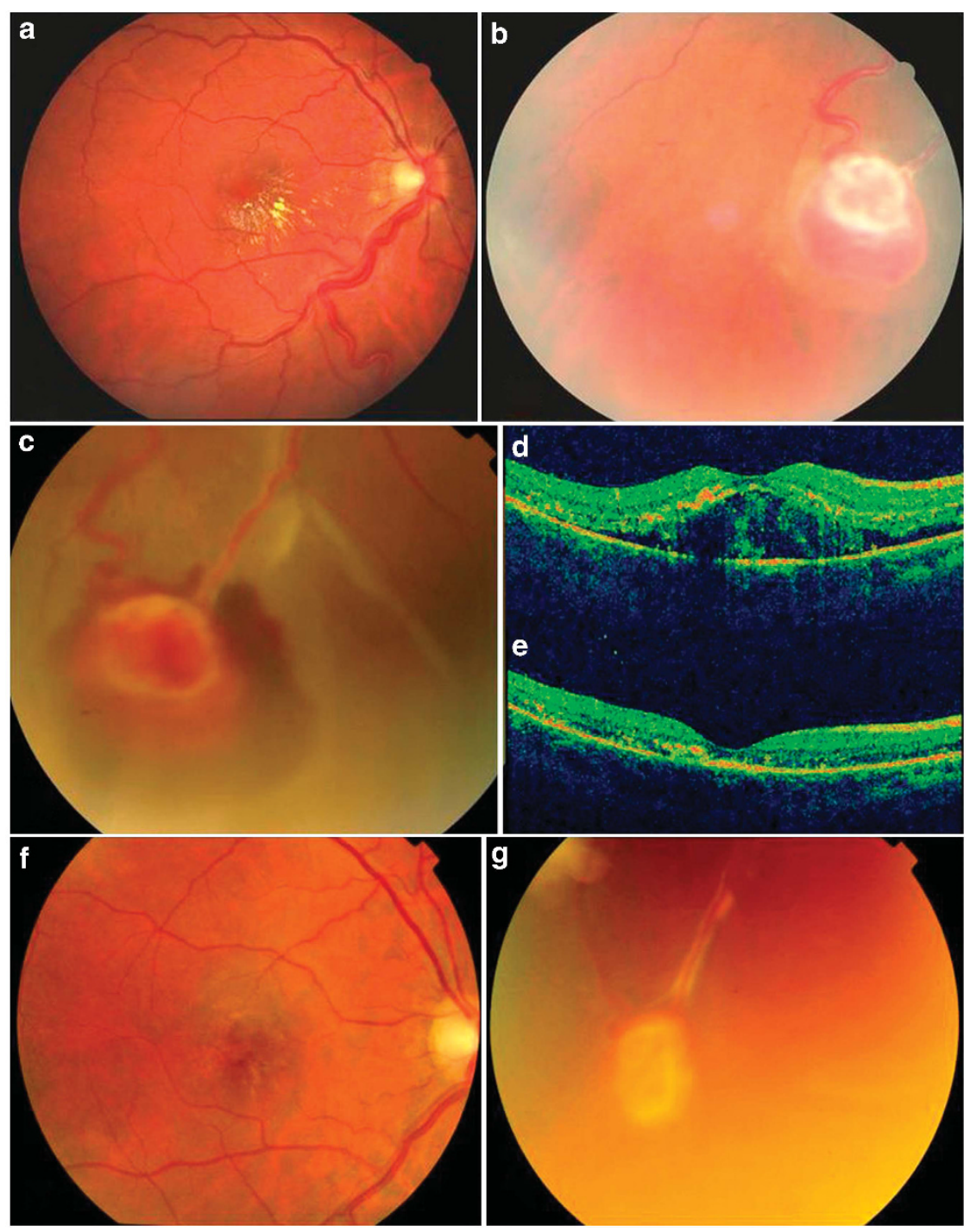

Figure 2 Color fundus (CF) photographs and spectral domain optical coherence tomography (SDOCT) foveal B scans of the right eye of a 32-year-old female (patient 5) (a-e). (a) CF photograph of the retinal periphery shows a RCH (before PDT). (b) CF photograph of the retinal periphery shows an area of exudative RD and an area of subretinal hemorrhage (1 week after PDT). (c) CF photograph of the retinal periphery shows a regressed RCH (9 months after PDT). (d) SDOCT foveal B scan suggestive of a serous macular detachment (1 week after PDT). (e) SDOCT foveal B scan shows resolved serous macular detachment (2 weeks after PDT). (f) CF photograph of the macula shows regressed exudates with RPE alteration in the fovea. $(\mathrm{g}) \mathrm{CF}$ photograph of the retinal periphery shows a regressed RCH (9 months after PDT).

responsiveness or unresponsiveness of the tumor to treatment remains to be elucidated.

\section{Summary}

\section{What was known before}

- That the treatment of the $\mathrm{RCH}$ is challenging, particularly the juxtapapillary variant.

\section{What this study adds}

- PDT for RCH can be effective in treating macular edema, however, this may not translate into improvement in VA in all cases.

\section{Conflict of interest}

The authors declare no conflict of interest.

\section{References}

1 Singh AD, Nouri M, Shields CL, Shields JA, Perez N. Treatment of retinal capillary haemangioma. Ophthalmology 2002; 109: 1799-1806.

2 Schmidt-Erfurth UM, Kusserow C, Barbazetto IA, Laqua H. Benefits and complications of photodynamic therapy of papillary capillary haemangiomas. Ophthalmology 2002; 109(7): 1256-1266. 
3 Atebara NH. Retinal capillary haemangioma treated with verteporfin photodynamic therapy. Am J Ophthalmol 2002; 134: 788-790.

4 Aaberg Jr TM, Aaberg Sr TM, Martin DF, Gilman JP, Myles R. Three cases of large retinal capillary haemangiomas treated with verteporfin and photodynamic therapy. Arch Ophthalmol 2005; 123(3): 328-332.

5 Golshevsky JR, O'Day J. Photodynamic therapy in the management of juxtapapillary capillary haemangiomas. Clin Experiment Ophthalmol 2005; 33(5): 509-512.

6 Szabo A, Gehl Z, Seres A. Photodynamic (verteporfin) therapy for retinal capillary haemangioma, with monitoring of feeder and draining blood vessel diameters. Acta Ophthalmol Scand 2005; 83(4): 512-513.

7 Ziemssen F, Voelker M, Inhoffen W, Bartz-Schmidt KU, Gelisken F. Combined treatment of a juxtapapillary retinal capillary haemangioma with intravitreal bevacizumab and photodynamic therapy. Eye 2007; 21(8): 1125-1126.

8 Sachdeva R, Dadgostar H, Kaiser PK, Sears JE, Singh AD. Verteporfin photodynamic therapy of six eyes with retinal capillary haemangioma. Acta Ophthalmol 2010; 88(8): 334-340.

9 Wong YM, Jalil A, Mathews J, Stanga PE. Exudative retinal detachment following photodynamic therapy for retinal capillary haemangioma. Can J Ophthalmol 2010; 21: 1125-1126.

10 Baba T, Kitahashi M, Kubota-Taniai M, Oshitari T, Yamamoto S. Subretinal hemorrhage after photodynamic therapy for juxtapapillary retinal capillary haemangioma. Case Rep Ophthalmol 2011; 2: 134-139.

11 Mennel S, Meyer CH, Callizo J. Combined intravitreal antivascular endothelial growth factor (Avastin) and photodynamic therapy to treat retinal juxtapapillary capillary haemangioma. Acta Ophthalmol 2010; 88(5): 610-613.

12 Mennel S, Meyer CH, Eggarter F, Peter S. Transient serous retinal detachment in Claasic and Occult choroidal neovascularization after photodynamic therapy. Am J Ophthalmol 2005; 140: 758-760. 